\title{
NOMINATA DOS PARECERISTAS
}

A Revista Conjuntura Global agradece a todos os pesquisadores que gentilmente aceitaram contribuir com a elaboração de pareceres através do sistema de avaliação cega pelos pares (double blind review). Abaixo listamos os pareceristas que contribuíram com a revista em 2020.

Acácio Telechi

Alexandre Ramos Coelho

Alexsandro Eugenio Pereira

Augusto Dall'Agnol

Bárbara Lopes Campos

Bruna Coelho Jaeger

Bruno Hendler

Bruno Rafael Maciel Trentini

Cairo Gabriel Borges Junqueira

Camila Feix Vidal

Carlos Magno Vasconcellos

Caroline Cordeiro Viana Silva

Claudia Santos

Claudia Stephan

Devlin Biezus

Evelise Zampier Da Silva

Fabiane Cristina Silva Mesquita

Fábio Santino Bussmann

Felipe Bernardo Estre

Felipe Florencio

Gabriel Fernandes Pimenta

Gabriela Angelico

Guilherme Frizzera

Gustavo Glodes Blum

Isaias Albertin Moraes
Ítalo de Paula Casemiro

Larissa Naves de Deus

Laura Lammerhirt

Leonardo Mèrcher

Ludmila Culpi

Mateus Albuquerque

Matheus de Abreu Costa Souza

Meryl Thiel

Murilo Gomes da Costa

Naiara Almeida Alcantara

Natali Hoff

Octávio Forti Neto

Paula Gomes Moreira

Rachel Silva da Rocha Coutinho

Ramon Blanco

Renan Holanda Montenegro

Ricardo Abrate Luigi Junior

Roberto Rodolfo Georg Uebel

Tadeu Morato Maciel

Thais Lemos Ribeiro

Thales Carvalho

Thalita Franciely de Melo Silva

Thiago Assunção

Victória Almeida 